\title{
Centrally Banked Cryptocurrencies
}

\author{
George Danezis \\ University College London \\ g.danezis@ucl.ac.uk
}

\author{
Sarah Meiklejohn \\ University College London \\ s.meiklejohn@ucl.ac.uk
}

\begin{abstract}
Current cryptocurrencies, starting with Bitcoin, build a decentralized blockchain-based transaction ledger, maintained through proofs-of-work that also serve to generate a monetary supply. Such decentralization has benefits, such as independence from national political control, but also significant limitations in terms of computational costs and scalability. We introduce RSCoin, a cryptocurrency framework in which central banks maintain complete control over the monetary supply, but rely on a distributed set of authorities, or mintettes, to prevent double-spending. While monetary policy is centralized, RSCoin still provides strong transparency and auditability guarantees. We demonstrate, both theoretically and experimentally, the benefits of a modest degree of centralization, such as the elimination of wasteful hashing and a scalable system for avoiding doublespending attacks.
\end{abstract}

\section{INTRODUCTION}

Bitcoin [25], introduced in 2009, and the many alternative cryptocurrencies it has inspired (e.g., Litecoin and Ripple), have achieved enormous success: financially, in November 2015, Bitcoin held a market capitalization of 4.8 billion USD and 30 cryptocurrencies held a market capitalization of over 1 million USD. In terms of visibility, cryptocurrencies have been accepted as a form of payment by an increasing number of international merchants, such as the 150,000 merchants using either Coinbase or Bitpay as a payment gateway provider.

Recently, major financial institutions such as JPMorgan Chase [28] and Nasdaq [27] have announced plans to develop blockchain technologies. The potential impacts of cryptocurrencies have now been acknowledged even by government institutions: the European Central Bank anticipates their "impact on monetary policy and price stability" [8]; the US Federal Reserve their ability to provide a "faster, more secure and more efficient payment system" [5]; and the UK Treasury vowed to "support innovation" [13] in this space. This is unsurprising, since the financial settlement systems currently in use by central banks (e.g., CHAPS, TARGET2, and Fedwire) remain relatively expensive and - at least behind the scenes - have high latency and are stagnant in terms of innovation.

Despite their success, existing cryptocurrencies suffer from a number of limitations. Arguably the most troubling one is their poor scalability: the Bitcoin network (currently by far

Permission to freely reproduce all or part of this paper for noncommercial purposes is granted provided that copies bear this notice and the full citation on the first page. Reproduction for commercial purposes is strictly prohibited without the prior written consent of the Internet Society, the first-named author (for reproduction of an entire paper only), and the author's employer if the paper was prepared within the scope of employment.

NDSS '16, 21-24 February 2016, San Diego, CA, USA

Copyright 2016 Internet Society, ISBN 1-891562-41-X

http://dx.doi.org/10.14722/ndss.2016.23187 the most heavily used) can handle at most 7 transactions per second $^{1}$ and faces significant challenges in raising this rate much higher, ${ }^{2}$ whereas PayPal handles over 100 and Visa handles on average anywhere from 2,000 to 7,000. This lack of scalability is ultimately due to its reliance on broadcast and the need to expend significant computational energy in proofsof-work - by some estimates [26, Chapter 5], comparable to the power consumption of a large power plant - in order to manage the transaction ledger and make double-spending attacks prohibitively expensive. Alternative cryptocurrencies such as Litecoin try to distribute this cost, and Permacoin [24] tries to repurpose the computation, but ultimately neither of these solutions removes the costs. A second key limitation of current cryptocurrencies is the loss of control over monetary supply, providing little to no flexibility for macroeconomic policy and extreme volatility in their value as currencies.

Against this backdrop, we present RSCoin, a cryptocurrency framework that decouples the generation of the monetary supply from the maintenance of the transaction ledger. Our design decisions were largely motivated by the desire to create a more scalable cryptocurrency, but were also inspired by the research agenda of the Bank of England [3], and the question of "whether central banks should themselves make use of such technology to issue digital currencies." Indeed, as Bitcoin becomes increasingly widespread, we expect that this will be a question of interest to many central banks around the world.

RSCoin's radical shift from traditional cryptocurrencies is to centralize the monetary supply. Every unit of a particular currency is created by a particular central bank, making cryptocurrencies based on RSCoin significantly more palatable to governments. Despite this centralization, RSCoin still provides the benefit over existing (non-crypto) currencies of a transparent transaction ledger, a distributed system for maintaining it, and a globally visible monetary supply. This makes monetary policy transparent, allows direct access to payments and value transfers, supports pseudonymity, and benefits from innovative uses of blockchains and digital money.

Centralization of the monetary authority also allows RSCoin to address some of the scalability issues of fully decentralized cryptocurrencies. In particular, as we describe in Section VI, the central bank delegates the authority of validating transactions to a number of other institutions that we call mintettes (following Laurie [19]). Since mintettes areunlike traditional cryptocurrency miners - known and may ultimately be held accountable for any misbehavior, RSCoin supports a simple and fast mechanism for double-spending detection. As described in Section $\mathrm{V}$, we adapt a variant

\footnotetext{
${ }^{1}$ http://en.bitcoin.it/wiki/Scalability

${ }^{2}$ http://en.bitcoin.it/wiki/Blocksize_debate
} 
of Two-Phase Commit, optimized to ensure the integrity of a transaction ledger. Thus, we achieve a significantly more scalable system: the modest experimental testbed that we describe in Section V-D2 (consisting of only 30 mintettes running a basic Python implementation of our consensus mechanism), can process over 2,000 transactions per second, and performance scales linearly as we increase the number of mintettes. Most transactions take less than one second to clear, as compared to many minutes in traditional cryptocurrency designs.

Beyond scalability, recent issues in the Bitcoin network have demonstrated that the incentives of miners may be misaligned, ${ }^{3}$ and recent research suggests that this problem-namely, that miners are incentivized to produce blocks without fully validating all the transactions they contain - is only exacerbated in other cryptocurrencies [21]. We therefore additionally discuss in Section VI-B1 how mintettes may collect fees for good service, and how such fees may be withheld from misbehaving or idle mintettes; our hope is that this framework can lead to a more robust set of incentives. In a real deployment of RSCoin, we furthermore expect mintettes to be institutions with an existing relationship to the central bank, such as commercial banks, and thus to have some existing incentives to perform this service.

The ultimate goal for RSCoin is to achieve not only a scalable cryptocurrency that can be deployed and whose supply can be controlled by one central bank, but a framework that allows any central bank to deploy their own cryptocurrency. In fact, there is interest [2] to allow other entities to not only issue instruments that hold value (such as shares and derivative products), but to furthermore allow some visibility into transactions concerning them. With this in mind, we discuss in Section VII-C what is needed to support some notion of interoperability between different deployments of RSCoin, how different currencies can be exchanged in a transparent and auditable way, and how various considerations - such as a pair of central banks that, for either security or geopolitical reasons, do not support each other - can be resolved without fragmenting the global monetary system. We also discuss other extensions and optimizations in Section VII.

\section{RELATED WORK}

Much of the research on cryptocurrencies either has analyzed the extent to which existing properties (e.g., anonymity and fairness) are satisfied or has proposed new methods to improve certain features. We focus on those works that are most related to the issues that we aim to address, namely stability and scalability.

The work on these two topics has been largely attack-based, demonstrating that even Bitcoin's heavyweight mechanisms do not provide perfect solutions. As demonstrated by Eyal and Sirer [9] and Garay et al. [10], an attacker can temporarily withhold blocks and ultimately undermine fairness. Babaioff et al. [1] argued that honest participation in the Bitcoin network was not sufficiently incentivized, and Johnson et al. [14] and Laszka et al. [18] demonstrated that in fact some participants might be incentivized to engage in denial-of-service attacks against each other. Karame et al. [15] and Rosenfeld [31]

\footnotetext{
${ }^{3}$ https://bitcoin.org/en/alert/2015-07-04-spv-mining
}

\begin{tabular}{lccc|c}
\hline & CC & e-cash & Bitcoin & RSCoin \\
\hline Double-spending & online & offline & online & online \\
Money generation & C & C & D & C \\
Ledger generation & C & n.a. & D & D* \\
Transparent & no & no & yes & yes \\
Pseudonymous & no & yes & yes & yes \\
\hline
\end{tabular}

TABLE I: How existing approaches (credit cards, cryptographic e-cash, and Bitcoin) and how RSCoin compare in terms of the properties they provide. Double-spending refers to the way the system detects double-spending (i.e., as it happens or after the fact). C stands for centralized, D for decentralized, and D* for distributed.

consider how an adversary might take advantage of both mining power and the network topology to execute a double-spending attack. Finally, Gervais et al. [11] looked at the structure of mining pools, the rise of SPV clients, and the privileged rights of Bitcoin developers and concluded that Bitcoin was far from achieving full decentralization. On the positive side, Kroll et al. [17] analyzed a simplified model of the Bitcoin network and concluded that Bitcoin is (at least weakly) stable.

In terms of other constructions, the work perhaps most related to our own is Laurie's approach of designated authorities [19]. This solution, however, does not describe a consensus mechanism or consider a centralized entity responsible for the generation of a monetary supply. The RSCoin framework is also related to the approaches adopted by Ripple and Stellar, in that the underlying consensus protocols $[22,32]$ used by all three sit somewhere between a fully decentralized setting - in which proof-of-work-based "Nakamoto consensus" [6] has thus far been adopted almost unilaterally — and a fully centralized setting (in which consensus is trivial). Within this space, RSCoin makes different trust assumptions and thus ends up with different features: both the Stellar and Ripple consensus protocols avoid a central point of trust, but at the cost of needing a broadcast channel (because the list of participants is not fixed a priori) and requiring servers to be in constant direct communication, whereas our use of a central bank - which, leaving aside any scalability benefits, is ultimately one of the main goals of this work - allows us to avoid both broadcast channels (because the set of mintettes is known and thus users can contact them directly) and direct communication between mintettes.

Finally, our approach borrows ideas from a number of industrial solutions. In particular, our two-layered approach to the blockchain is in part inspired by the Bitcoin startup Factom, and our consensus mechanism is in part inspired by Certificate Transparency [20]. In particular, RSCoin, like Certificate Transparency, uses designated authorities and relies on transparency and auditability to ensure integrity of a ledger, rather than full trust in a central party.

\section{BACKGROUND}

In this section, we present a brief background on Bitcoin and traditional cryptocurrencies, and introduce some relevant notation. Since RSCoin adopts properties of other online payment systems, such as those of credit cards and cryptographic 
e-cash, we highlight some of the advantages and disadvantages of each of these approaches in Table I.

\section{A. The Bitcoin protocol}

Bitcoin is a decentralized cryptocurrency introduced in a whitepaper in 2008 [25] and deployed on January 3 2009. Since then, Bitcoin has achieved success and has inspired a number of alternative cryptocurrencies (often dubbed "altcoins") that are largely based on the same blockchain technology. The novelty of this blockchain technology is that it fulfills the two key requirements of a currency - the generation of a monetary supply and the establishment of a transaction ledger - in a completely decentralized manner: a global peer-to-peer network serves both to generate new units of currency and to bear witness to the transfer of existing units from one party to another through transaction broadcast and computational proofof-work protocols.

To highlight the differences between RSCoin and fully decentralized cryptocurrencies such as Bitcoin, we sketch the main operations and entities of these blockchain-based currencies; for a more comprehensive overview, we refer the reader to Bonneau et al. [6]. Briefly, users can generate signing keypairs and use the public key as a pseudonym or address in which to store some units of the underlying cryptocurrency. To transfer the value stored in this address to the address of another user, he creates a transaction, which is cryptographically signed using the secret key associated with this address. (More generally, transactions can transfer value from $m$ input addresses to $n$ output addresses, in which case the transaction must be signed by the secret keys associated with each of the input addresses.)

Once a user has created a transaction, it is broadcast to his peers in the network, and eventually reaches miners. A miner seals the transaction into the global ledger by including it in a pool of transactions, which she then hashes - along with some metadata and, crucially, a nonce - to attempt to produce a hash below a target value (defined by the difficulty of the network). Once a miner is successful in producing such a hash, she broadcasts the pool of transactions and its associated hash as a block. Among the metadata for a block is a reference to the previously mined block, allowing the acceptance of the miner's block into the blockchain to be signaled by the broadcast of another block with a reference to hers (or, in practice, many subsequent blocks). Miners are incentivized by two rewards: the collection of optional fees in individual transactions, and a system-specific mining reward (e.g., as of November 2015, Bitcoin's mining reward of 25 BTC). These rewards are collected in a special coin generation transaction that the miner includes in her block's pool of transactions. Crucially, blocks serve to not only generate the monetary supply (via the mining rewards included in each block), but also to provide a partial ordering for transactions: transactions in one block come before transactions included in any block further along the blockchain. This allows all users in the network to eventually impose a global (partial) ordering on transactions, and thus thwart double-spending by maintaining a list of unspent transaction outputs and validating a transaction only if its input addresses appear in this list.

What we have described above is the typical way of explaining Bitcoin at a high level, but we mention that in reality, bitcoins are not "stored" in an address or "sent"; instead, the sender relinquishes control by broadcasting a transaction that re-assigns to the recipient's address the bitcoins previously associated with that of the sender. An input to a transaction is thus not an address but a (signed) script that specifies an index in a previous transaction in which some bitcoins were received; this address identifier uniquely identifies one particular usage of an address, which becomes important as addresses are reused. In what follows, we thus frequently use the notation for an address and for a transaction-index pair interchangeably.

\section{B. Notation}

We denote a hash function as $H(\cdot)$ and a signature scheme as the tuple (Sig.KeyGen, Sig.Sign, Sig.Verify), where these algorithms behave as follows: via $(p k, s k) \stackrel{\$}{\leftarrow} \operatorname{Sig} . \operatorname{KeyGen}\left(1^{\lambda}\right)$ one generates a signing keypair; via $\sigma \stackrel{\$}{\leftarrow} \operatorname{Sig}$. Sign $(s k, m)$ one generates a signature; and via $0 / 1 \leftarrow \operatorname{Sig}$. Verify $(p k, m, \sigma)$ one verifies a signature on a message.

We use addr to denote an address; this is identical to a public key $p k$ in terms of the underlying technology, ${ }^{4}$ but we use the separate term to disambiguate between usage in a transaction (where we use addr) and usage as a signing key (where we use $p k$ ). We use $\operatorname{tx}\left(\left\{\operatorname{addr}_{i}\right\}_{i} \stackrel{n}{\rightarrow}\left\{\operatorname{addr}_{j}\right\}_{j}\right)$ to denote a transaction in which $n$ units of currency are sent from $\left\{\operatorname{addr}_{i}\right\}_{i}$ to $\left\{\operatorname{addr}_{j}\right\}_{j}$. Each usage of an address addr can be uniquely identified by the tuple addrid $=\left(\mathrm{tx}, \operatorname{index}_{\mathrm{tx}}(\mathrm{addr}), v\right)$, where $t x$ is the hash of the transaction in which it received some value $v$, and index $(\mathrm{addr})$ is the index of addr in the list of outputs. When we use these address identifiers later on, we occasionally omit information (e.g., the value $v$ ) if it is already implicit or unnecessary.

\section{AN OVERVIEW OF RSCOIN}

In this section, we provide a brief overview of RSCoin, which will be useful for understanding both its underlying consensus algorithm (presented in Section V) and the composition of the system as a whole (presented in Section VI).

At a high level, RSCoin introduces a degree of centralization into the two typically decentralized components of a blockchain-based ledger: the generation of the monetary supply and the constitution of the transaction ledger. In its simplest form, the RSCoin system assumes two structural entities: the central bank, a centralized entity that ultimately has complete control over the generation of the monetary supply, and a distributed set of mintettes (following Laurie [19]) that are responsible for the maintenance of the transaction ledger. The interplay between these entities - and an overview of RSCoin as a whole — can be seen in Figure 1.

Briefly, mintettes collect transactions from users and collate them into blocks, much as is done with traditional cryptocurrencies. These mintettes differ from traditional cryptocurrency miners, however, in a crucial way: rather than performing some computationally difficult task, each mintette is simply authorized by the central bank to collect transactions. In RSCoin, this authorization is accomplished by a PKI-type functionality, meaning the central bank signs the public key of

\footnotetext{
${ }^{4} \mathrm{Or}$, as in Bitcoin, it may be some hashed version of the public key.
} 


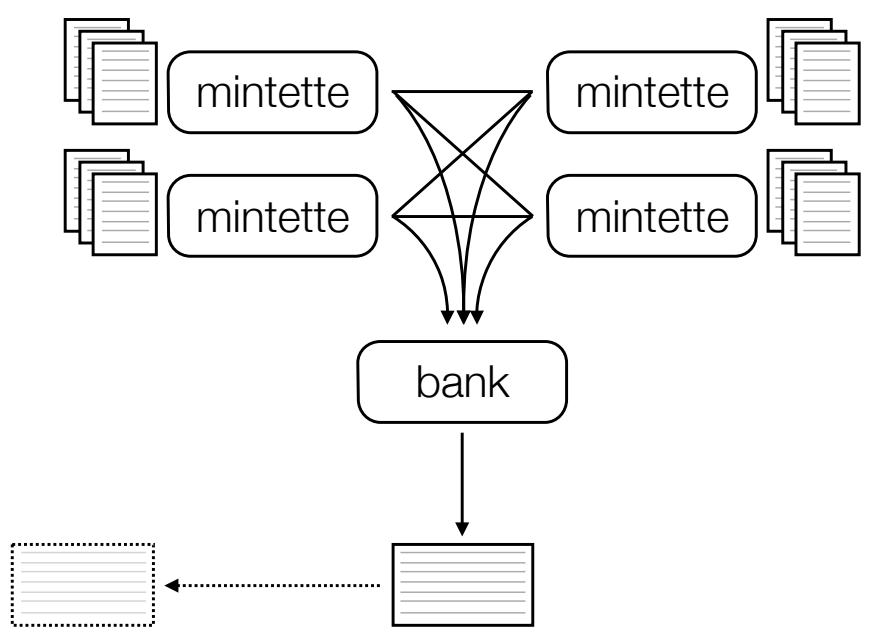

Fig. 1: The overall structure of RSCoin. Each mintettes maintains a set of lower-level blocks, and (possibly) communicates with other mintettes (either directly or indirectly). At some point, the mintettes send these blocks to the central bank, which produces a higher-level block. It is these higher-level blocks that form a chain and that are visible to external users.

the mintette, and each lower-level block must contain one of these signatures in order to be considered valid. We refer to the time interval in which blocks are produced by mintettes as an epoch, where the length of an epoch varies depending on the mintette. Because these blocks are not ultimately incorporated into the main blockchain, we refer to them as lower-level blocks. Mintettes are collectively responsible for producing a consistent ledger, and thus to facilitate this process they communicate internally throughout the course of an epoch - in an indirect manner described in Section $\mathrm{V}$ - and ultimately reference not only their own previous blocks but also the previous blocks of each other. This means that these lower-level blocks form a (potentially) cross-referenced chain.

At the end of some longer pre-defined time interval called a period, the mintettes present their blocks to the central bank, which merges these lower-level blocks to form a consistent history in the form of a new block. This higher-level block is what is ultimately incorporated into the main blockchain, meaning a user of RSCoin need only keep track of higherlevel blocks. (Special users wishing to audit the behavior of the mintettes and the central bank, however, may keep track of lower-level blocks, and we describe in Section V-C ways to augment lower-level blocks to improve auditability.)

Interaction with RSCoin can thus be quite similar to interaction with existing cryptocurrencies, as the structure of its blockchain is nearly identical, and users can create new pseudonyms and transactions in the same way as before. In fact, we stress that RSCoin is intended as a framework rather than a stand-alone cryptocurrency, so one could imagine incorporated techniques from various existing cryptocurrencies in order to achieve various goals. For example, to ensure privacy for transactions, one could adapt existing cryptographic techniques such as those employed by Zerocoin [23], Zerocash [4], Pinocchio Coin [7], or Groth and Kohlweiss [12]. As these goals are somewhat orthogonal to the goals of this paper, we

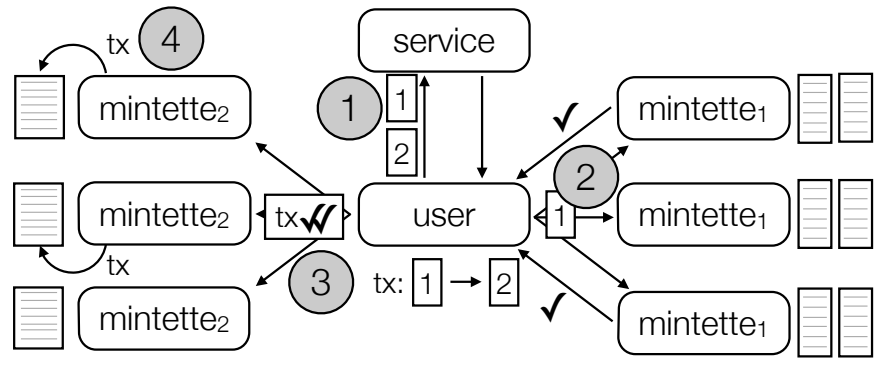

Fig. 2: The proposed protocol for validating transactions; each mintette $\mathrm{m}_{i}$ is an owner of address $i$. In (1), a user learns the owners of each of the addresses in its transaction. In (2), the user collects approval from a majority of the owners of the input addresses. In (3), the user sends the transaction and these approvals to the owners of the transaction identifier. In (4), some subset of these mintettes add the transaction to their blocks.

leave a comprehensive exploration of how privacy-enhancing and other techniques can be combined with RSCoin as an interesting avenue for future work.

\section{ACHIEVING CONSENSUS}

In the previous section, we described how mintettes send so-called "lower-level blocks" to the central bank at the end of a period. In this section, we describe a consensus protocol by which these blocks can already be made consistent when they are sent to the central bank, thus ensuring that the overall system remains scalable by allowing the central bank to do the minimal work necessary.

As described in the introduction, one of the major benefits of centralization is that, although the generation of the transaction ledger is still distributed, consensus on valid transactions can be reached in a way that avoids the wasteful proofsof-work required by existing cryptocurrencies. In traditional cryptocurrencies, the set of miners is neither known nor trusted, meaning one has no choice but to broadcast a transaction to the entire network and rely on proof-of-work to defend against Sybil attacks. Since our mintettes are in fact authorized by the central bank, and thus both known and - because of their accountability - trusted to some extent, we can avoid the heavyweight consensus requirement of more fully decentralized cryptocurrencies and instead use an adapted version of TwoPhase Commit (2PC), as presented in Figure 2. A generic consensus protocol, ensuring total ordering of transactions, is not necessary for double-spending prevention; instead, a weaker property - namely that any transaction output features as a transaction input in at most one other transaction - is sufficient. RSCoin builds its consensus protocol for doublespending prevention based on this insight.

We begin by described a threat model for the consensus protocol before going on to present a basic protocol that achieves consensus on transactions (Section V-B), an augmented protocol that allows for auditability of both the mintettes and the central bank (Section V-C), and a performance evaluation (Section V-D). 


\section{A. Threat model and security}

We always assume that the central bank is honestalthough we describe in Section VI-B1 ways to detect certain types of misbehavior on the part of the bank - and that the underlying cryptography is secure; i.e., no parties may violate the standard properties offered by the hash function and digital signature. Honest mintettes follow the protocols as specified, whereas dishonest mintettes may behave arbitrarily; i.e., they may deviate from the prescribed protocols, and selectively or broadly ignore requests from users. Finally, honest users create only valid transactions (i.e., ones in which they own the input addresses and have not yet spent their contents), whereas dishonest users may try to double-spend or otherwise subvert the integrity of RSCoin.

We consider two threat models. Our first threat model assumes that each transaction is processed by a set of mintettes with an honest majority; this is different from assuming that a majority of all mintettes are honest, as we will see in our description of transaction processing in Section V-B. Our second threat model assumes that no mintette is honest, and that mintettes may further collude to violate the integrity of RSCoin. This is a very hostile setting, but we show that some security properties still hold for honest users. Additionally, we show that mintettes that misbehave in certain ways can be detected and ultimately held accountable, which may serve as an incentive to follow the protocols correctly.

In the face of these different adversarial settings, we try to satisfy at least some of the following key integrity properties:

- No double-spending: Each output address of a valid transaction will only ever be associated with the input of at most one other valid transaction.

- Non-repudiable sealing: The confirmation that a user receives from a mintette - which promises that a transaction will be included in the ledgercan be used to implicate that mintette if the transaction does not appear in the next block.

* Timed personal audits: A user can, given access to the lower-level blocks produced within a period, ensure that the implied behavior of a mintette matches the behavior observed at the time of any previous interactions with that mintette.

* Universal audits: Anyone with access to the lower-level blocks produced within a period can audit all transactions processed by all mintettes. In particular, mintettes cannot retroactively modify, omit, or insert transactions in the ledger.

* Exposed inactivity: Anyone with access to the lower-level blocks produced within a period can observe any mintette's substantial absence from participation in the $2 \mathrm{PC}$ protocol. (In particular, then, a mintette cannot retroactively act to claim transaction fees for services not provided in a timely manner.)

To see how to satisfy these security properties, we first present our basic consensus protocol in Section V-B, and then present in Section V-C ways to augment this protocol to achieve auditability. We then prove that at least some subset of these security properties can be captured in both our threat models, and that exposure may disincentive mintettes from violating those that we cannot capture directly.

\section{B. A basic consensus protocol}

To begin, the space of possible transaction identifiers is divided in a random fashion so that each mintette $\mathrm{m}$ is responsible for some subset, or "shard." For reliability and security, each shard is covered by (potentially) multiple mintettes, and everyone is aware of the owner(s) of each.

We use owners(addrid) to denote the set of mintettes responsible for addrid. Recall that addrid $=(\mathrm{tx}, i, v)$, where tx specifies the transaction in which addr, at sequential output $i$, received value $v$. We map each addrid to a shard using tx, which can be done by hashing a canonical representation of the transaction. As a result, all input addrid in a transaction may have different owners (because the addresses may have appeared as an output in different transactions), but all output addrid have the same owner (because they are all appearing as an output in the same transaction). For simplicity, we therefore use the notation owners $\left(S_{\text {out }}\right)$ below (where $S_{\text {out }}$ is the list of output addresses for a transaction).

In each period, each mintette $\mathrm{m}$ is responsible for maintaining two lists concerning only the addrid (and indirectly the transactions tx) it owns: a list of unspent transaction outputs, denoted utxo, and two lists of transactions seen thus far in the period, denoted pset and txset respectively (the former is used to detect double-spending, and the latter is used to seal transactions into the ledger). The utxo list is of the form addrid $\mapsto($ addr,$v)$, where (addrid $\mapsto($ addr,$v)) \in$ utxo indicates that addrid had not acted as an input address at the start of the period but has since sent value $v$ to addr and (addrid $\mapsto(\perp, \perp)) \in$ utxo indicates that addrid has not yet spent its contents. The pset list is of the form addrid $\mapsto t x$, where (addrid $\mapsto \mathrm{tx}) \in$ pset indicates that addrid has acted as an input address in transaction tx. We assume that each mintette starts the period with an accurate utxo list (i.e., all transactions within the mintette's shard in which the outputs have not yet been spent) and with an empty pset.

At some point in the period, a user creates a transaction. The user ${ }^{5}$ can now run Algorithm V.1. ${ }^{6}$

In the first phase, the user asks the relevant mintettes to "vote" on the transaction; i.e., to decide if its input addresses have not already been used, and thus certify that no doublespending is taking place. To do this, the user can compute the owners for each input address, and send the transaction information to these mintettes, who each run Algorithm V.2. We omit for simplicity the formal description of an algorithm CheckTx that, on input a transaction, checks that the basic structure of the transaction is valid; i.e., that the collective input value is at least equal to the collective output value, that the input address identifiers point to valid previous transactions, and that the signatures authorizing previous transaction outputs to be spent are valid.

\footnotetext{
${ }^{5}$ We refer to the user here and in the sequel, but in practice this can all be done by the underlying client, without any need for input from the (human) user.

${ }^{6}$ All algorithms are assumed to be executed atomically and sequentially by each party, although as we demonstrate in Section V-D2, implementing them using optimistic locking is possible to increase parallelism and efficiency.
} 

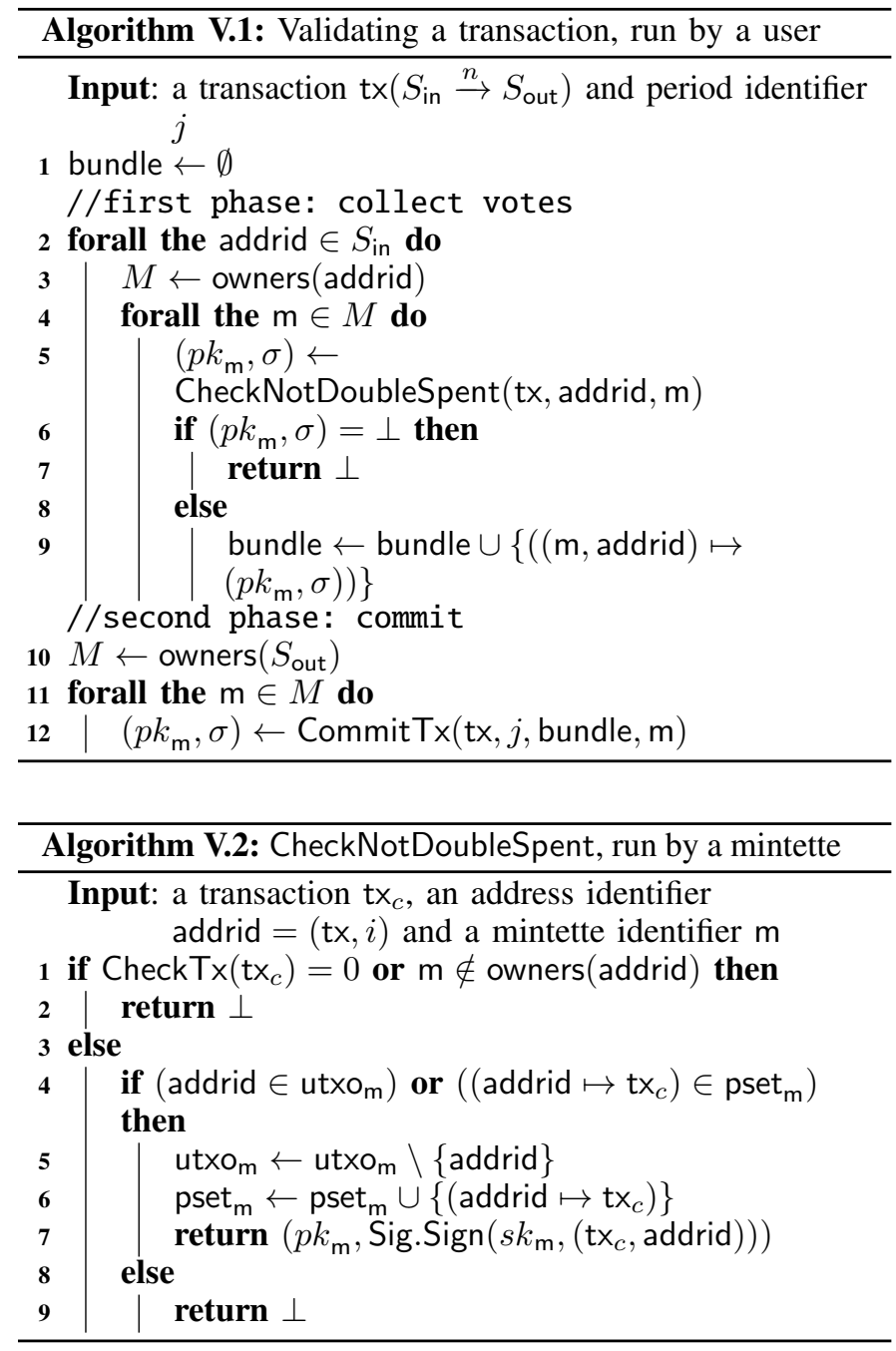

Briefly, in Algorithm V.2 the mintette first checks if the current transaction is valid and if the address is within its remit, and returns $\perp$ otherwise. It then proceeds if the address identifier either has not been spent before (and thus is in utxo), or if it has already been associated with the given transaction (and thus the pair is in pset). In those cases, it removes the address identifier from utxo and associates it with the transaction in pset; these actions are idempotent and can be safely performed more than once. The mintette then returns a signed acknowledgment to the user. If instead another transaction appears in pset associated with the address identifier, then the address is acting as an input in two different transactions - i.e., it is double-spending - and the mintette returns $\perp$.

At the end of the first phase, an honest user thus will have received some signatures (representing 'yes' votes) from the owners of the input addresses of the new transaction. Users should check the signatures returned by these mintettes and immediately return a failure if any is invalid. Once the user has received signatures from the majority of owners for each input, she can now send the transaction, coupled with a "bundle of evidence" (consisting of the signatures of the input mintettes) to represent its validity, to the owners of the output addresses (who, recall, are the same for all output addresses). These mintettes then run Algorithm V.3.

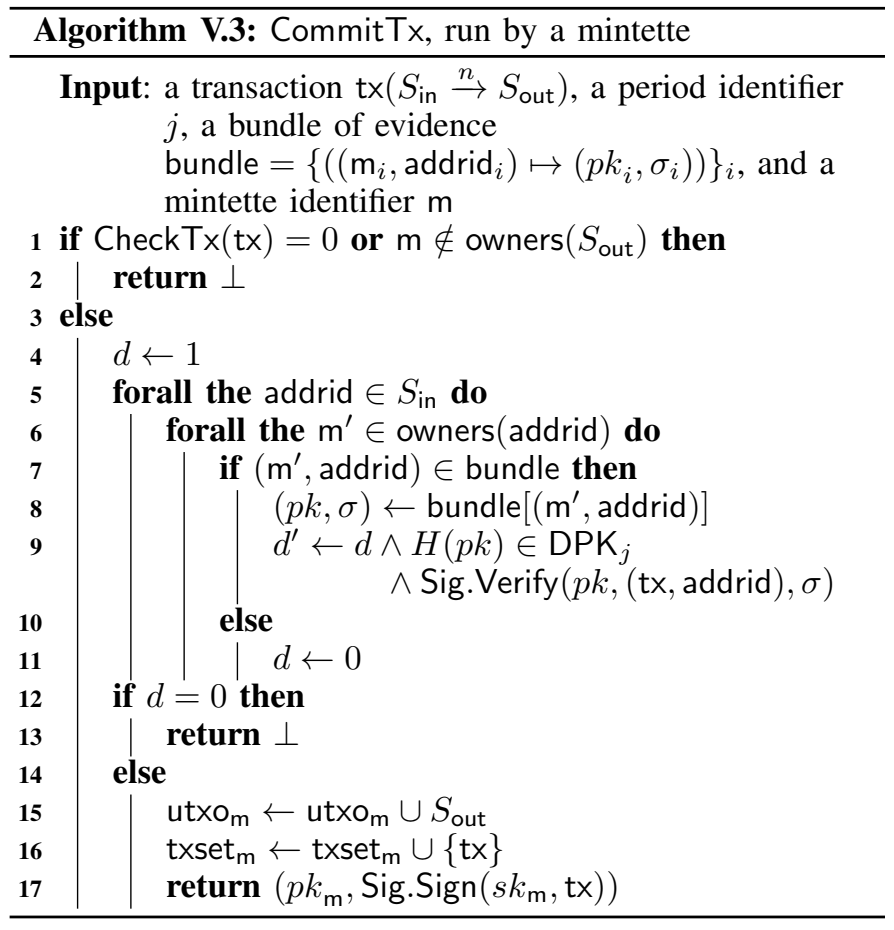

In Algorithm V.3, a mintette first checks the transaction and whether it falls within its remit. The mintette then checks the bundle of evidence by verifying that all — or, in practice, a majority - of mintettes associated with each input are all included, that the input mintettes were authorized to act as mintettes in the current period, and that their signatures verify. If these checks pass and the transaction has not been seen before, then the mintette adds all the output addresses for the transaction to its utxo list and adds the transaction to txset. The mintette then sends to the user evidence that the transaction will be included in the higher-level block (which a user may later use to implicate the mintette if this is not the case).

At the end of the period, all mintettes send txset to the central bank, along with additional information in order to achieve integrity, which we discuss in the next section.

a) Security: In our first threat model, where all transactions are processed by a set of mintettes with honest majority, it is clear that (1) no double-spending transactions will be accepted into txset by honest mintettes, and (2) the confirmation given to a user in Line 17 of Algorithm V.3 can be wielded by the user as evidence that the mintette promised to seal the transaction. Thus, in our first threat model - in which all transactions are processed by a set of mintettes with honest majority - the first and second integrity properties in Section $\mathrm{V}-\mathrm{A}$ are already satisfied by our basic consensus protocol.

b) Communication overhead: Importantly, all communication between the mintettes is done indirectly via the user (using the bundles of evidence), so in particular there is no direct communication between them. This allows for a low communication overhead for the mintettes, especially with respect to existing systems such as Bitcoin and Ripple/Stellar (in which the respective miners and servers must be in constant communication), which facilitates - as we will see in 
Section V-D2 - the scalability and overall performance benefits of RSCoin.

\section{Achieving auditability}

While our basic consensus mechanism already achieves some of our desired integrity properties (at least in our weaker threat model), it is still not clear that it provides any stronger notions of integrity, or that it provides any integrity in a more hostile environment. To address this limitation, we present in this section a way to augment both the lower-level blocks discussed in Section VI-A and the basic consensus mechanism. At a high level, a mintette now maintains a high-integrity log that highlights both its own key actions, as well as the actions of those mintettes with whom it has indirectly interacted (i.e., from whom it has received signatures, ferried through the user, in the process of committing a transaction).

In more detail, each mintette maintains a log of absolutely ordered actions along with their notional sequence number. Actions may have one of three types: Query, Commit and CloseEpoch. The Query action signals an update to pset as a result of an input address being assigned to a new transaction (Line 6 of Algorithm V.2), so for this action the log includes the new transaction. The Commit action signals an update to utxo and txset as a result of receiving a new valid transaction (lines 15 and 16 of Algorithm V.3, respectively), so for this action the log includes the transaction and its corresponding bundle of evidence.

To facilitate the CloseEpoch action, each mintette stores not only the $\log$ itself but also a rolling hash chain; i.e., a head that acts as a witness to the current state of the log, so $h_{\text {seq }}=H\left(a_{\text {seq }} \| h_{\text {seq-1 }}\right)$, where $a_{\text {seq }}$ is the log entry of the action and $h_{s e q-1}$ is the previous head of the chain.

To share this witness, mintettes include a signed head in every message they emit; i.e., in line 7 of Algorithm V.2 and line 17 of Algorithm V.3, the mintette $m$ computes $\sigma \stackrel{\$}{\leftarrow} \operatorname{Sig} \cdot \operatorname{Sign}\left(s k_{\mathrm{m}},\left(\mathrm{tx}_{c}\right.\right.$, addrid, $\left.h, s e q\right)$ (where $h$ is the head of its chain) rather than $\sigma \stackrel{\$}{\leftarrow} \operatorname{Sig} . \operatorname{Sign}\left(s k_{\mathrm{m}},\left(\mathrm{tx}_{c}\right.\right.$, addrid $\left.)\right)$, and outputs $\left(p k_{\mathrm{m}}, \sigma, h, s e q\right)$. Now that mintettes are potentially aware of each others' $\log$, the CloseEpoch action - which, appropriately, marks the end of an epoch - includes in the log the heads of the other chains of which the mintette is aware, along with their sequence number. This results in the head of each mintette's chain depending on the latest known head of both its own and other chains; we refer to this phenomenon as cross-hashing (which, in effect, implements a cryptographic variant of vector clocks [30]).

We can now argue that these augmented lower-level blocks provide sufficient insight into the actions of the mintettes that stronger notions of integrity can be achieved. In particular, we have the following lemma:

Lemma V.1. In both of our threat models, the augmented consensus protocol outlined above provides timed personal audits, universal audits, and exposed inactivity (as defined in Section $V$-A).

Proof: (Informal.) To prove that our protocol provides timed personal audits, observe that if the log reported by any mintette (or equivalently its hash at any log position) forks at any point from the record of a user or other mintette, then the signed head of the hash chain serves as evidence that the log is different. To remain undetected, the mintette must therefore provide users with the signed head of a hash chain that is a prefix of the actual hash chain it will report. Both the Query and Commit messages leading to a signed hash, however, modify the action log. Providing an outdated hash thus would not contain the latest action, so again there is evidence that such an action should have been recorded (in the form of the signed response to the message that should prompt the action), which also incriminates the mintette. Thus a mintette that does not wish to be detected and incriminated may only refrain from responding to requests requiring actions that would change its $\log$.

To prove that our protocol provides universal audits and exposed inactivity, we first note that, despite the lack of synchronization between mintettes within periods, we can detect when an action is committed to a mintette $\log$ a 'significant time' after another action. This is due to the fact that the second message of the $2 \mathrm{PC}$ protocol that users send to mintettes carries the hash heads from all input mintettes involved. This forms a low-degree random graph with good expansion properties, and we expect that in a short amount of time mintettes will have hash chains dependent on the hash chains of all other mintettes. Thus, if two actions are separated by a sufficiently long period of time, it is extremely likely that a head dependent on the first action has propagated to a super-majority of other mintettes. Checking this property allows us to detect which came first with very high probability. Using this observation, everyone may audit claims that a mintette contributed to an action (e.g., processing the first query of the $2 \mathrm{PC}$ protocol for a valid transaction) in a timely fashion, by using the process above to detect whether the claimed action from the mintette is or is not very likely to have come after the same action was committed by all other mintettes concerned.

Finally, RSCoin makes the key security assumption that all shards are composed of an honest majority of mintettes. This is not quite the same as assuming an overall honest majority of mintettes, but it can be related to the more traditional assumption that each mintette behaves honest with some probability, as we demonstrate in the following lemma:

Lemma V.2. Given a fraction of a corrupt mintettes, the probability that $y$ shards, composed each of $Q$ mintettes, all have an honest majority is

$$
\operatorname{Pr}[\text { secure }]=F\left(\frac{Q-1}{2} ; Q ; \alpha\right)^{y},
$$

where $F(k ; N ; p)$ is the cumulative distribution function of a binomial distribution over a population of size $N$ with a probability of success $p$.

Proof: The probability that a single shard composed from random mintettes has an honest majority is directly the cumulative distribution $\rho=F\left(\frac{Q-1}{2} ; Q ; \alpha\right)$. Since security requires an honest majority across all shards we get $\operatorname{Pr}[$ secure $]=\rho^{y}$.

This lemma demonstrates that the higher the number of shards, the lower the probability that all of them will be secure (i.e., covered by an honest majority of mintettes). Thus, we 
recommend fixing the number of shards, on the basis of load balancing requirements, to the smallest practical number. A mapping can then be defined between the address space and the shards by simply partitioning equally the space of address identifiers amongst them. For a given total number of mintettes $M$, the minimal number of shards of size $Q$ that should be used is $\lfloor M / Q\rfloor$.

\section{Performance}

1) Theoretical analysis: Looking back at the algorithms in Section V-B, we can get at least a theoretical estimate of the communication and computational complexity of the system. Denote by $T$ the set of transactions that are generated per second; by $Q$ the number of mintettes that own each address; and by $M$ the number of total mintettes.

For a transaction with $m$ inputs and $n$ outputs, a user sends and receives at most $m Q$ messages in the first phase of the 2PC protocol (line 5 of Algorithm V.1) and sends and receives at most $Q$ messages in the second phase (line 12). For the user, each transaction thus requires at most $2(m+1) Q$ messages.

In terms of the communication complexity per mintette, we assume that each mintette receives a proportional share of the total transactions, which is ensured as the volume of transactions grow, by the bank allocating shards of equal sizes to all mintettes. Then the work per mintette is

$$
\frac{\sum_{\mathrm{tx} \in T} 2\left(m_{\mathrm{tx}}+1\right) Q}{M} .
$$

In particular, this scales infinitely: as more mintettes are added to the system, the work per mintette decreases (in a linear fashion) and eventually goes to zero.

2) Experimental analysis: To verify these performance estimates and to measure the latency a typical user would experience to confirm a transaction, we implemented the basic consensus mechanism presented in Section V-B and measured its performance on a modest cluster hosted on Amazon's Elastic Compute (EC2) infrastructure. Our implementation consists of 2458 lines of Python code: 1109 lines define the core transaction structure, cryptographic processing, and $2 \mathrm{PC}$ protocols as a Twisted service and client; 780 lines are devoted to unit and timing tests; and 569 lines use the Fabric framework to do configuration, deployment management (DevOps), live testing, and visualizations. Both the clients and the mintettes are implemented as single-threaded services following a reactor pattern. All cryptographic operations use the OpenSSL wrapper library petlib, and we instantiate the hash function and digital signature using SHA-256 and ECDSA (over the NISTP224 curve, as optimized by Käsper [16]) respectively. The implementation and all configuration and orchestration files necessary for replicating our results are available under a BSD license.

Our experimental setup consisted of 30 mintettes, each running on an Amazon EC2 t2.micro instance in the EU (Ireland) data center (for reference, each cost $\$ 0.014$ per hour as of August 2015). We assigned three mintettes to each shard of the transaction space, so a quorum of at least two was required for the $2 \mathrm{PC}$. A different set of 25 servers on the same data center was used for stress testing and estimating the peak throughput in terms of transactions per second. Each of those

\begin{tabular}{lrr}
\hline Benchmark & \multicolumn{1}{c}{$\mu\left(\mathrm{s}^{-1}\right)$} & \multicolumn{1}{c}{$\sigma$} \\
\hline Hash & $1,017,384.86$ & $41,054.93$ \\
Sign & $17,043.63$ & 2316.40 \\
Verify & 4651.20 & 89.84 \\
\hline Check tx & 3585.02 & 95.17 \\
Query msg & 1358.31 & 120.20 \\
Commit msg & 1006.49 & 31.66 \\
\hline
\end{tabular}

TABLE II: Micro-benchmarks at the mintettes

test machines issued 1000 transactions consisting of two inputs and two outputs. For wide area networking latency experiments we used a residential broadband cable service and an Ubuntu 14.02.2 LTS Linux VM running on a 64-bit Windows 7 laptop with a $2.4 \mathrm{GHz}$ i7-4700MQ processor and 16GB RAM.

Table II reports the mean rate and the standard deviation of key operations we rely on for RSCoin. ${ }^{7}$ Hash, Sign and Verify benchmark the number of basic cryptographic operations each mintette can perform per second (including the overhead of our library and Python runtime).

For the other benchmarks, we consider a single transaction with one input and two outputs (we observe that as of September $2014,53 \%$ of Bitcoin transactions had this structure, so this is a reasonable proxy for real usage). The check tx benchmark then measures the rate at which a mintette can parse and perform the cryptographic checks associated with this transaction. This involves a single signature check, and thus its difference from the Sign benchmark largely represents the overhead of parsing and of binary conversion in Python. Guided by this benchmark, we chose to represent ECDSA public keys using uncompressed coordinates due to orders-of-magnitude slowdowns when parsing keys in compressed form.

The query msg and commit msg benchmarks measure the rate at which each mintette can process the first and second message of the $2 \mathrm{PC}$ respectively for this transaction. These include full de-serialization, checks from persistent storage of the utxo, cryptographic checks, updates to the utxo, signing, and serialization of responses. These benchmarks guided our design towards not synchronizing to persistent storage the utxo before each response, and relying instead on the quorum of mintettes to ensure correctness (a design philosophy similar to RAMCloud [29]). Persisting to storage before responding to each request slowed these rates by orders of magnitude.

Figure 3 illustrates the latency a client would experience when interacting with the mintettes. Figure $3 a$ illustrates the experiments with client machines within the data center, and point to an intrinsic delay due to networking overheads and cryptographic checks of less than 0.5 seconds. This includes both phases of the $2 \mathrm{PC}$.

Over a wide area network the latency increases (Figure 3b), but under the conditions tested, the latency is still usually well under a second for the full 2PC and all checks. We note that no shortcuts were implemented: for each transaction, all three mintettes for each input were contacted and expected to

\footnotetext{
${ }^{7}$ All measurements were performed on a single thread on a single core, using a reactor pattern where networking was necessary.
} 


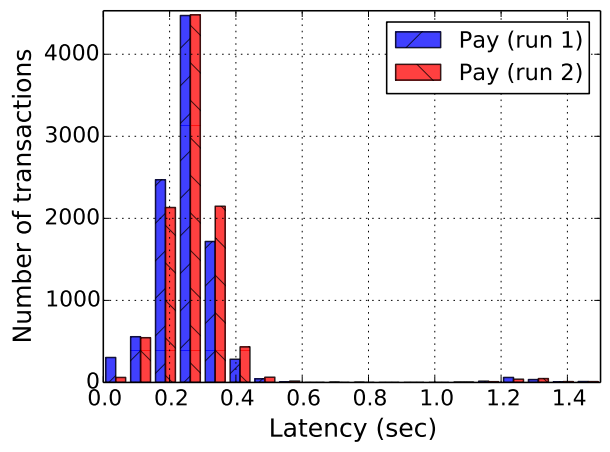

(a) Local area network (EC2)

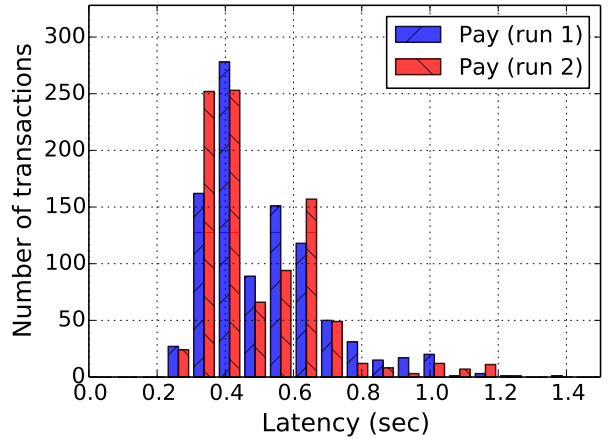

(b) Wide area network (Broadband)

Fig. 3: Latency, in seconds, to perform the $2 \mathrm{PC}$ to validate a payment for a transaction with freshly issued coins as inputs (run 1), and transactions with two arbitrary previous transactions as inputs (run 2).

respond in the first phase, and all three mintettes responsible for the new transaction were contacted and have to respond in the second phase. In reality, only a majority need to respond before concluding each phase, and this may reduce latency further.

Figure 4 plots the throughput of the system as we increase the number of mintettes from 2 to 30, under the load of 25 synthetic clients, each pushing 1000 transactions. As expected, when fewer than three mintettes are available the throughput is roughly flat (fewer than 400 transactions per second), as both phases of the 2PC need to contact all mintettes. Once more than the minimum of three mintettes are available the load is distributed across them: the first phase need to access at most six mintettes (three for each of the two transaction inputs), and the second phase at most three mintettes. This load per transaction is independent of the number of mintettes and as a result the throughput scales linearly, as predicted in Section V-D1. After the initial three mintettes, each new mintette adds approximately 66 additional transactions per second to the capacity of the system.

The gap between the micro-benchmarks relating to the message processing for the two phases (1358.31 $\mathrm{s}^{-1}$ and $1006.49 \mathrm{~s}^{-1}$ respectively) and the rate of transactions observed under end-to-end conditions (approximately $400 \mathrm{~s}^{-1}$ ) indicates

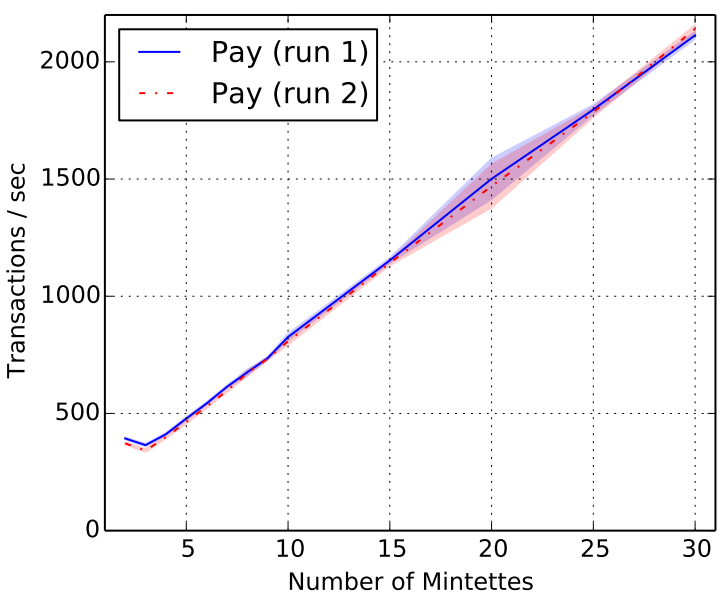

Fig. 4: Throughput ( $90^{\text {th }}$ percentile and standard error), in transactions per second, as a function of the number of mintettes, for transactions with two freshly issued coins as inputs (run 1) and transactions with two arbitrary previous transactions as inputs (run 2).

that at this point bandwidth, networking, or the interconnection with the process are scaling bottlenecks for single mintettes. In particular no pipelining was implemented as part of the client (although the mintettes support it) and thus every request initiates a fresh TCP connection, with the slowdowns and resource consumption on the hosts that this entails.

\section{THE RSCOIN SYSTEM}

With our consensus protocol in place, we now describe the structure of RSCoin, focusing on the interaction between the mintettes and the central bank, and on the overall parameters and properties of the system. We first describe the structure and usage of RSCoin (Sections VI-A and VI-B) and then address considerations that arise in how to allocate fees to mintettes (Section VI-B1); overlay RSCoin on top of an existing cryptocurrency like Bitcoin (Section VI-B2); incentivize mintettes to follow the consensus protocol and present a collectively consistent ledger to the central bank (Section VI-C); and set concrete choices for various system parameters (Section VI-D).

\section{A. Lower-level blocks}

A lower-level block produced by a mintette $\mathrm{m}$ within period $\mathrm{d}_{i}$ looks like $\mathrm{b}=(h$, txset, $\sigma$, mset), where $h$ is a hash, txset is a collection of transactions, and $\sigma$ is a signature from the mintette that produced this block. The fourth component mset specifies the cross-chain property of lower-level blocks (recall from Section V-C that mintettes may reference each others' blocks) by identifying the hashes of the other previous blocks that are being referenced.

Denote by $p k_{\text {bank }}$ the bank's public key and by $\mathrm{DPK}_{i}$ the set of mintettes authorized by the bank in the previous higher-level block $\mathrm{B}_{\text {bank }}^{(i-1)}$ (as described in Section VI-B), and define otherblocks $\leftarrow h_{1}\|\ldots\| h_{n}$ for mset $=\left(h_{1}, \ldots, h_{n}\right)$. Assuming the block $\mathrm{b}$ is produced in epoch ${ }_{j}$, to check that $\mathrm{b}$ is valid one then checks that 
1) $\quad h=H\left(h_{\text {bank }}^{(i-1)}\left\|h_{j-1}^{(\mathrm{m})}\right\|\right.$ otherblocks $\|$ txset $)$,

2) Sig.Verify $\left(p k_{\mathrm{m}}, h, \sigma\right)=1$,

3) $\left(p k_{\mathrm{m}}, \sigma_{\text {bank }}^{(\mathrm{m})}\right) \in \mathrm{DPK}_{i}$ for some $\sigma_{\text {bank }}^{(\mathrm{m})}$, and

4) $\quad \operatorname{Sig} . V_{\text {erify }}\left(p k_{\text {bank }},\left(p k_{\mathrm{m}}, \operatorname{period}_{i}\right), \sigma_{\text {bank }}^{(\mathrm{m})}\right)=1$.

To form a lower-level block, a mintette uses the transaction set txset it has formed throughout the epoch (as described in Section V-B) and the hashes $\left(h_{1}, \ldots, h_{n}\right)$ that it has received from other mintettes (as ferried through the "bundle of evidence" described in Section V-C) and creates mset $\leftarrow\left(h_{1}, \ldots, h_{n}\right)$, otherblocks $\leftarrow h_{1}\|\ldots\| h_{n}$, $h \leftarrow H\left(h_{\text {bank }}^{(i-1)}\left\|h_{j-1}^{(\mathrm{m})}\right\|\right.$ otherblocks $\|$ txset $), \quad$ and $\sigma \stackrel{\$}{\leftarrow}$ $\operatorname{Sig} . \operatorname{Sign}\left(s k_{\mathrm{m}}, h\right)$.

\section{B. Higher-level blocks}

The higher-level block that marks the end of period ${ }_{i}$ looks like $\mathrm{B}_{\text {bank }}^{(i)}=\left(h\right.$, txset, $\left.\sigma, \mathrm{DPK}_{i+1}\right)$, where these first three values are similar to their counterparts in lower-level blocks (i.e., a hash, a collection of transactions, and a signature), and the set $\mathrm{DPK}_{i+1}$ contains pairs $\left(p k_{\mathrm{m}}, \sigma_{\text {bank }}^{(\mathrm{m})}\right)$; i.e., the public keys of the mintettes authorized for period ${ }_{i+1}$ and the bank's signatures on the keys.

To check that a block is valid, one checks that

1) $h=H\left(h_{\text {bank }}^{(i-1)} \|\right.$ txset $)$,

2) $\operatorname{Sig.Verify}\left(p k_{\text {bank }}, h, \sigma\right)=1$, and

3) Sig.Verify $\left(p k_{\text {bank }},\left(p k_{\mathrm{m}}, \operatorname{period}_{i+1}\right), \sigma_{\text {bank }}^{(\mathrm{m})}\right)=1$ for all $\left(p k_{\mathrm{m}}, \sigma_{\text {bank }}^{(\mathrm{m})}\right) \in \mathrm{DPK}_{i+1}$.

To form a higher-level block, the bank must collate the inputs it is given by the mintettes, which consist of the lowerlevel blocks described above and the action logs described in Section V-C. To create a consistent transaction set txset, a vigilant bank might need to look through all of the transaction sets it receives to detect double-spending, remove any conflicting transactions, and identify the mintette(s) responsible for including them. As this would require the bank to perform work proportional to the number of transactions (and thus somewhat obviate the reason for mintettes), we also consider an optimistic approach in which the bank relies on the consensus protocol in Section $\mathrm{V}$ and instead simply merges the individual transaction sets to form txset. The bank then forms $h \leftarrow H\left(h_{\text {bank }}^{(i-1)} \|\right.$ txset $)$, $\sigma \stackrel{\$}{\leftarrow} \operatorname{Sig} \cdot \operatorname{Sign}\left(p k_{\text {bank }}, h\right)$, and creates the set of authorized mintettes using a decision process we briefly discuss below and in Section VI-C.

1) Coin generation and fee allocation: In addition to this basic structure, each higher-level block could also contain within txset a special coin generation transaction and an allocation of fees to the mintettes that earned them in the previous period. Semantically, the coin generation could take on the same structure as in Bitcoin; i.e., it could be a transaction $\mathrm{tx}\left(\emptyset \stackrel{n}{\rightarrow} \mathrm{addr}_{\text {bank }}\right)$, where $\operatorname{addr}_{\text {bank }}$ is an address owned by the bank, and fees could be allocated using a transaction $\mathrm{t} \times\left(\mathrm{addr}_{\text {bank }} \stackrel{f}{\rightarrow} \mathrm{addr}_{\mathrm{m}}\right)$, where $f$ represents the fees owed to $\mathrm{m}$. The interesting question is thus not how central banks can allocate fees to mintettes, but how it decides which mintettes have earned these fees. In fact, the provided action logs allow the central bank to identify active and live mintettes and allocate fees to them appropriately.
This mechanism (roughly) works as follows. The central bank keeps a tally of the mintettes that were involved in certifying the validity of input addresses; i.e., those that replied in the first phase of the consensus protocol. The choice to reward input mintettes is deliberate: in addition to providing a direct incentive for mintettes to respond in the first phase of the protocol, it also provides an indirect incentive for mintettes to respond in the second phase, as only a transaction output that is marked as unspent can later be used as an input (for which the mintette can then earn fees). Thus, rewarding input mintettes provides incentive to handle a transaction throughout its lifetime.

The action logs also play a crucial role in fee allocation. In particular, the "exposed inactivity" security property from Section V-C prevents an inactive mintette from becoming active at a later time and claiming that it contributed to previous transactions, as an examination of the action logs can falsify such claims. Additionally, if fee allocation is determined on the basis of a known function of the action logs, anyone with access to the action logs can audit the actions of the central bank.

Finally, we mention that although the logs are sent only to the central bank, the expectation is that the central bank will publish these logs to allow anyone to audit the system. As we assume the central bank is honest, this does not present a problem, but in a stronger threat model in which less trust were placed in the central bank, one might instead attempt to adopt a broadcast system for distributing logs (with the caveat that this approach introduces significantly higher latency). In such a setting, anyone with access to the logs could verify not only the actions of the mintettes, but could also replay these actions to compare the ledger agreed upon by the mintettes and the ledger published by the bank; this would allow an auditor to ensure that the bank was not engaging in misbehavior by, e.g., dropping transactions.

2) A simplified block structure: The above description of higher-level blocks (and the previous description of lower-level blocks) contains a number of additional values that do not exist in the blocks of existing cryptocurrencies, making RSCoin somewhat incompatible with their semantics. To demonstrate that RSCoin can more strongly resemble these cryptocurrencies, we briefly describe a way of embedding these additional values into the set of transactions.

Rather than include the set $\mathrm{DPK}_{i+1}$, the bank could instead store some units of currency in a master address addr bank and include in txset $_{i}$ a transaction $\mathrm{t} \times\left(\operatorname{addr}_{\text {bank }} \stackrel{n_{p k}}{\longrightarrow} \operatorname{addr}_{\text {bank }}^{(i+1)}\right)$, where $\operatorname{addr}_{\text {bank }}^{(i+1)}$ is an address specific to period ${ }_{i+1}$. The bank could then include in $\operatorname{txset}_{i}$ a transaction $\operatorname{tx}\left(\operatorname{addr}_{\text {bank }}^{(i+1)} \stackrel{n_{\mathrm{m}}}{\longrightarrow}\right.$ $p k_{\mathrm{m}}$ ) for each mintette $\mathrm{m}$ authorized for period ${ }_{i+1}$. Now, to check the validity of a particular lower-level block, one could check that such a transaction was included in the previous higher-level block.

\section{Incentivizing mintettes}

One might naturally imagine that this structure, as currently described, places the significant burden on the central bank of having to merge the distinct blocks from each mintette into a consistent history. By providing appropriate incentives, however, 
we can create an environment in which the presented ledger is in fact consistent before the bank even sees it. If mintettes deviate from the expected behavior then, as we described in Section VI-B1, they can be held accountable and punished accordingly (e.g., not chosen for future periods or not given any fees they have earned).

Section VI-B1 describes one direct incentive for mintettes to collect transactions, which is fees. As we described in Section VI-B1, mintettes are rewarded only for active participation, so that an authorized mintette needs to engage with the system in order to earn fees. Section VI-B2 describes another direct incentive, which is the authorization of mintettes by the central bank. For semantic purposes, the value $n_{\mathrm{m}}$ used to authorize each mintette for the next period could be arbitrarily small. As an incentive, however, this value could be larger to directly compensate the mintettes for their services.

Finally, we expect that the central bank could be a national or international entity that has existing relationships with, e.g., commercial banks. There thus already exist strong business incentives and regulatory frameworks for such entities to act as honest mintettes.

\section{Setting system parameters}

As described, the system is parameterized by a number of variables, such as the length of epochs, the length of a period, and the number of mintettes. The length of an epoch for an individual mintette is entirely dependent on the rate at which it processes transactions (as described in detail in Section V-C). Mintettes that process more transactions will therefore have shorter epochs than ones that do so less frequently. There is no limit on how short an epoch can be, and the only upper limit is that an epoch cannot last longer than a period.

It might seem desirable for periods to be as short as possible, as ultimately a transaction is sealed into the official ledger only at the end of a period. To ease the burden on the bank, however, it is also desirable to have longer periods, so that central banks have to intervene as infrequently as possible (and, as we describe in Section VII-A, so that central banks can potentially perform certain optimizations to reduce transaction bloat). In Section V-B, we described methods by which mintettes could "promise" (in an accountable way) to users that their transactions would be included, so that in practice near-instantaneous settlement can be achieved even with longer periods, so long as one trusts the mintette. Nevertheless, we do not expect periods to last longer than a day.

For the purposes of having a fair and competitive settlement process, it is desirable to have as many mintettes as possible; as we saw in Section V-D1, this is also desirable from a performance perspective, as the performance of the RSCoin system (measured in the rate of transactions processed) scales linearly with the number of mintettes. Adding more mintettes, however, also has the effect that they earn less in transaction fees, so these opposing concerns must be taken into account when settling on a concrete number (to give a very rough idea, one number that has been suggested [2] is 200).

\section{OPTIMIZATIONS AND EXTENSIONS}

In Sections V and VI, we presented a (relatively) minimal version of RSCoin, which allows us to achieve the basic integrity and scalability properties that are crucial for any currency designed to be used on a global level. Here, we briefly sketch some extensions that could be adopted to strengthen either of these properties, and leave a more detailed analysis of these or other solutions as interesting future research.

\section{A. Pruning intermediate transactions}

At the end of a period, the central bank publishes a higherlevel block containing the collection of transactions that have taken place in that time interval; it is only at this point that transactions are officially recorded in the ledger. Because mintettes provide evidence on a shorter time scale that a user's transaction is valid and will be included in the ledger, however, users might feel more comfortable moving currency multiple times within a period than in traditional cryptocurrencies (in which one must wait for one or several blocks to avoid possible double-spending).

It therefore might be the case that at the end of a period, the central bank sees not just individual transactions, but potentially multiple "hops" or even whole "chains" of transactions. To limit transaction bloat, the bank could thus prune these intermediate transactions at the end of the period, so that ultimately only the start and end points of the transaction appear in the ledger, in a new transaction signed by the central bank.

On its surface, this idea may seem to require a significant amount of trust in the central bank, as it could now actively modify the transaction history. The action logs, however, would reveal the changes that the bank had made and allow users to audit its behavior, but nevertheless the alterations that could be made would need be significantly restricted.

\section{B. Further incentives for honest behavior}

In addition to the existing incentives for honest behavior outlined in Sections VI-B1 and VI-C, mintettes could adopt a sort of proof-of-stake mechanism, in which they escrow some units of currency with the central bank and are allowed to collate only a set of transactions whose collective value does not exceed the escrowed value. If any issue then arises with the transactions produced by the mintette (e.g., it has accepted double-spending transactions), the central bank can seize the escrowed value and remove the double-spending transactions, so the mintette ultimately pays for this misbehavior out of its own pocket (and maybe even pays additional fines).

This mechanism as described is not fully robust (as in particular the mintette might accept many expenditures of the same unit of currency, not just two), but it does have an interesting effect on the length of periods. In particular, the length of earlier periods will necessarily be quite small, as mintettes will not have much capital to post. As mintettes accumulate stores of currency, however, periods can grow longer. This is a fairly natural process, as it also allows for a trial period in the beginning to ensure that authorized mintettes don't misbehave, and then for a more stable system as a set of trustworthy mintettes emerges.

\section{Multiple banks and foreign exchange}

In a global setting, one might imagine that each central bank could develop their own version of RSCoin; this would 
lead, however, to a landscape much the same as today's Bitcoin and the many altcoins it has inspired, in which multiple implementations of a largely overlapping structure lead to an infrastructure fragmentation: bugs are replicated across codebases and compatibility across different altcoins is artificially low.

An attractive approach is for different central banks to instead use the same platform, to prevent this fragmentation and to allow users to seamlessly store value in many different currencies. While this allows the currencies generated by different central banks to achieve some notion of interoperability, we still expect that different blockchains will be kept separate; i.e., a particular central bank does not - and should not - have to keep track of all transactions that are denominated in the currency of another central bank. (Mintettes, however, may choose to validate transactions for any number of central banks, depending on their business interests.)

While every central bank does not necessarily need to be aware of transactions denominated in the currency of another central bank, this awareness may at times be desirable. For example, if a user would like to exchange some units of one currency into another belonging to a central bank that is relatively known to and trusted by the first (e.g., exchange GBP for USD), then this should be a relatively easy process. The traditional approach is to simply go to a third-party service that holds units of both currencies, and then perform one transaction to send units of the first currency to the service, which will show up in the ledger of the first currency, and another transaction to receive units of the second currency, which will show up in the ledger of the second currency.

Although this is the approach by far most commonly adopted in practice (both in fiat currency and cryptocurrency markets), it has a number of limitations, first and foremost of which is that it is completely opaque: even an outside observer who is able to observe both ledgers sees two transactions that are not linked in any obvious way. One might naturally wonder, then, if a more transparent mechansim is possible, in which the currency exchange shows up as such in the ledger. We answer this question in the affirmative in Appendix A, in which we demonstrate a form of fair exchange.

Briefly, to achieve this fair exchange, we adapt a protocol to achieve atomic cross-chain trading, ${ }^{8}$ which provides a Bitcoincompatible way for two users to fairly exchange units of one currency for some appropriate units of another currency; i.e., to exchange currency in a way that guarantees that either the exchange is successful or both users end up with nothing (so in particular it cannot be the case that one user reclaims currency and the other does not). If one is less concerned about compatibility with Bitcoin, then a slightly simpler approach such as "pay on reveal secret" [33] could be adopted.

To fit our setting, in which central banks may want to maintain some control over which other currencies their currency is traded into and out of (and in what volume), we modify the existing protocol to require a third party to sign both transactions only if they are denominated in currencies that are viewed as "exchangeable" by that party. This serves to not only

\footnotetext{
${ }^{8}$ The clearest explanation of this for Bitcoin, by Andrew Miller, can be found at bitcointalk.org/index.php?topic $=193281 . \mathrm{msg} 3315031 \# \mathrm{msg} 3315031$.
}

signal the third party's blessing of the exchange, but also to bind the two transactions together across their respective blockchains. Our proposal of this protocol thus enables transparent exchanges that can be approved by a third party, but does not (and cannot) prevent exchanges from taking place without this approval. Importantly, however, an auditor can now - with access to both blockchains - observe the exchange.

\section{CONCLUSIONS}

In this paper, we have presented the first cryptocurrency framework, RSCoin, that provides the control over monetary policy that entities such as central banks expect to retain. By constructing a blockchain-based approach that makes relatively minimal alterations to the design of successful cryptocurrencies such as Bitcoin, we have demonstrated that this centralization can be achieved while still maintaining the transparency guarantees that have made (fully) decentralized cryptocurrencies so attractive. We have also proposed a new consensus mechanism based on $2 \mathrm{PC}$ and measured its performance, illustrating that centralization of some authority allows for a more scalable system to prevent double spending that completely avoids the wasteful hashing required in proof-of-work-based systems.

\section{ACKNOWLEDGEMENTS}

We thank Robleh Ali, Simon Scorer, Alex Mitchell, and John Barrdear from the Bank of England and Ben Laurie from Google for interesting discussions. We also thank our anonymous reviewers and our shepherd, Joseph Bonneau, for their helpful feedback. George Danezis is supported in part by EPSRC Grant EP/M013286/1 and Sarah Meiklejohn is supported in part by EPSRC Grant EP/M029026/1.

\section{REFERENCES}

[1] M. Babaioff, S. Dobzinski, S. Oren, and A. Zohar, "On Bitcoin and red balloons," SIGecom Exchanges, vol. 10, no. 3, pp. 56-73, 2011.

[2] Bank of England, Private communication, 2015.

[3] — , "One bank research agenda," 2015, www.bankofengland.co.uk/research/Documents/onebank/ discussion.pdf.

[4] E. Ben-Sasson, A. Chiesa, C. Garman, M. Green, I. Miers, E. Tromer, and M. Virza, "Zerocash: Decentralized anonymous payments from bitcoin," in 2014 IEEE Symposium on Security and Privacy, SP 2014, Berkeley, CA, USA, May 18-21, 2014. IEEE Computer Society, 2014, pp. 459-474. [Online]. Available: http://dx.doi.org/10.1109/SP.2014.36

[5] B. Bernanke, Nov. 2013, qz.com/148399/ben-bernankebitcoin-may-hold-long-term-promise/.

[6] J. Bonneau, A. Miller, J. Clark, A. Narayanan, J. A. Kroll, and E. W. Felten, "Research perspectives and challenges for Bitcoin and cryptocurrencies," in Proceedings of the IEEE Symposium on Security and Privacy, 2015.

[7] G. Danezis, C. Fournet, M. Kohlweiss, and B. Parno, "Pinocchio coin: building zerocoin from a succinct pairing-based proof system," in PETShop'13, Proceedings of the 2013 ACM Workshop on Language Support for Privacy-Enhancing Technologies, Co-located with CCS 2013, November 4, 2013, Berlin, Germany, 
M. Franz, A. Holzer, R. Majumdar, B. Parno, and H. Veith, Eds. ACM, 2013, pp. 27-30. [Online]. Available: http://doi.acm.org/10.1145/2517872.2517878

[8] European Central Bank, "Virtual currency schemes - a further analysis," Feb. 2015, www.ecb.europa.eu/pub/pdf/ other/virtualcurrencyschemesen.pdf.

[9] I. Eyal and E. G. Sirer, "Majority is not enough: Bitcoin mining is vulnerable," in Proceedings of Financial Cryptography 2014, 2014.

[10] J. Garay, A. Kiayias, and N. Leonardos, "The Bitcoin backbone protocol: Analysis and applications," in Proceedings of Eurocrypt 2015, 2015.

[11] A. Gervais, G. O. Karame, S. Capkun, and V. Capkun, "Is Bitcoin a decentralized currency?" IEEE Security \& Privacy, vol. 12, pp. 54-60, 2014.

[12] J. Groth and M. Kohlweiss, "One-out-of-many proofs: Or how to leak a secret and spend a coin," in Advances in Cryptology - EUROCRYPT 2015 - 34th Annual International Conference on the Theory and Applications of Cryptographic Techniques, Sofia, Bulgaria, April 26-30, 2015, Proceedings, Part II, ser. Lecture Notes in Computer Science, E. Oswald and M. Fischlin, Eds., vol. 9057. Springer, 2015, pp. 253-280. [Online]. Available: http://dx.doi.org/10.1007/978-3-662-46803-6_9

[13] HM Treasury, "Digital currencies: response to the call for information," Mar. 2015, www.gov.uk/government/uploads/system/uploads/ attachment_data/file/414040/digital_currencies_ response_to_call_for_information_final_changes.pdf.

[14] B. Johnson, A. Laszka, J. Grossklags, M. Vasek, and T. Moore, "Game-theoretic analysis of DDoS attacks against Bitcoin mining pools," in Workshop on Bitcoin Research, 2014.

[15] G. Karame, E. Androulaki, and S. Capkun, "Double-Spending Fast Payments in Bitcoin," in Proceedings of ACM CCS 2012, 2012.

[16] E. Käsper, "Fast elliptic curve cryptography in openssl," in Financial Cryptography and Data Security - FC 2011 Workshops, RLCPS and WECSR 2011, Rodney Bay, St. Lucia, February 28 - March 4, 2011, Revised Selected Papers, ser. LNCS, G. Danezis, S. Dietrich, and K. Sako, Eds., vol. 7126. Springer, 2011, pp. 27-39.

[17] J. A. Kroll, I. C. Davey, and E. W. Felten, “The economics of Bitcoin mining, or Bitcoin in the presence of adversaries," in Proceedings of WEIS 2013, 2013.

[18] A. Laszka, B. Johnson, and J. Grossklags, "When Bitcoin mining pools run dry: A game-theoretic analysis of the long-term impact of attacks between mining pools," in Workshop on Bitcoin Research, 2015.

[19] B. Laurie, "An efficient distributed currency," 2011, www.links.org/files/distributed-currency.pdf.

[20] — "Certificate transparency," Commun. ACM, vol. 57, no. 10 , pp. 40-46, 2014.

[21] L. Luu, J. Teutsch, R. Kulkarni, and P. Saxena, "Demystifying incentives in the consensus computer," in Proceedings of ACM CCS 2015, 2015, to appear.

[22] D. Mazières, "The Stellar consensus protocol: a federated model for Internet-level consensus," 2015, www.stellar.org/papers/stellar-consensus-protocol.pdf.

[23] I. Miers, C. Garman, M. Green, and A. D. Rubin, "Zerocoin: Anonymous distributed e-cash from bitcoin," in 2013 IEEE Symposium on Security and Privacy, SP
2013, Berkeley, CA, USA, May 19-22, 2013. IEEE Computer Society, 2013, pp. 397-411. [Online]. Available: http://dx.doi.org/10.1109/SP.2013.34

[24] A. Miller, A. Juels, E. Shi, B. Parno, and J. Katz, "Permacoin: Repurposing Bitcoin work for data preservation," in Proceedings of the IEEE Symposium on Security and Privacy, 2014.

[25] S. Nakamoto, "Bitcoin: A Peer-to-Peer Electronic Cash System," 2008, bitcoin.org/bitcoin.pdf.

[26] A. Narayanan, J. Bonneau, E. Felten, A. Miller, and S. Goldfeder, Bitcoin and cryptocurrency technologies. [Online]. Available: piazza.com/princeton/spring2015/btctech/resources

[27] Nasdaq, "Nasdaq launches enterprise-wide blockchain technology initiative," May 2015, www.nasdaq.com/pressrelease/nasdaq-launches-enterprisewide-blockchaintechnology-initiative-20150511-00485.

[28] D. O'Leary, V. D’Agostino, S. R. Re, J. Burney, and A. Hoffman, "Method and system for processing Internet payments using the electronic funds transfer network," Nov. 2013. [Online]. Available: www.google.com/patents/US20130317984

[29] J. K. Ousterhout, P. Agrawal, D. Erickson, C. Kozyrakis, J. Leverich, D. Mazières, S. Mitra, A. Narayanan, D. Ongaro, G. M. Parulkar, M. Rosenblum, S. M. Rumble, E. Stratmann, and R. Stutsman, "The case for ramcloud," Commun. ACM, vol. 54, no. 7, pp. 121-130, 2011.

[30] M. Raynal and M. Singhal, "Logical time: Capturing causality in distributed systems," IEEE Computer, vol. 29, no. 2, pp. 49-56, 1996.

[31] M. Rosenfeld, "Analysis of hashrate-based double-spending," Dec. 2012, bitcoil.co.il/Doublespend.pdf.

[32] D. Schwartz, N. Youngs, and A. Britto, "The Ripple protocol consensus algorithm," 2014, ripple.com/files/ripple_consensus_whitepaper.pdf.

[33] T. Young, "Atomic cross-chain exchange," 2014, upcoder.com/11/atomic-cross-chain-exchange/.

\section{APPENDIX}

In Section VII-C, we described a protocol for atomic trading of different currencies and outlined some of its features, such as allowing trade only across authorized currencies (as determined by some third party). Our formal protocol that achieves this fair exchange is presented in Figure 5.

Informally, if Alice and Bob wish to exchange $m$ units of currency $c_{1}$ for $n$ units of currency $c_{2}$, with the blessing of a third party Carol, then they each create two types of transactions: a "spend" transaction, in which the sender releases the units of currency to one of two addresses, and a "refund" transaction, in which the sender can reclaim the currency after a certain amount of time has passed. The two addresses in Alice's spend transactions are a "multi-signature" address from which funds can be released only with the signatures of Alice, Bob, and Carol, or Bob's address, from which he can spend the funds only with knowledge of the pre-image of some hash $H(x)$. Her refund transaction then sends the currency back to Alice's address if signatures are provided by all three parties, and if an appropriate amount of time $t_{1}$ has elapsed since the spend transaction was accepted into the blockchain. Similarly, Bob's 


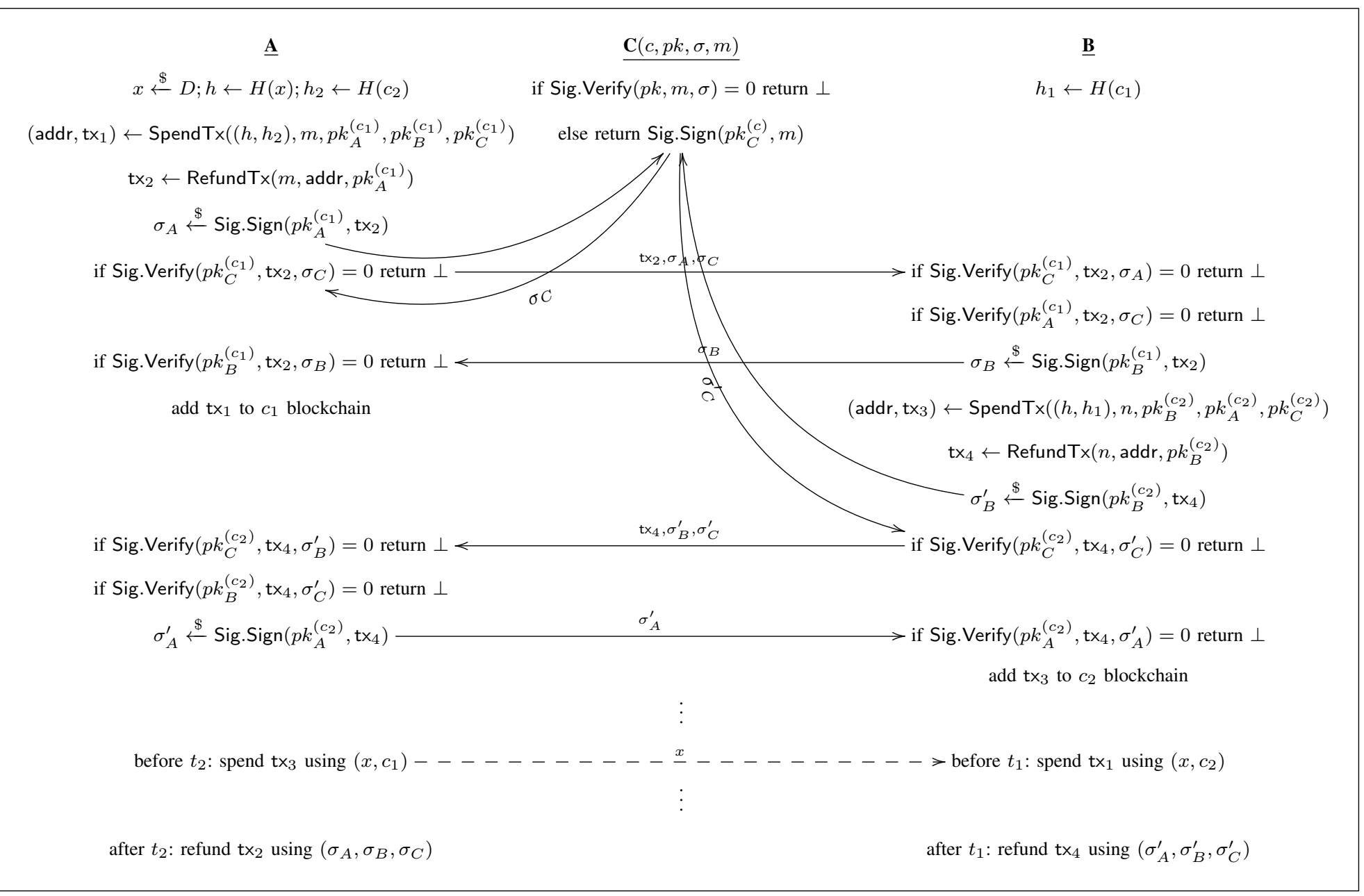

Fig. 5: A method for $A$ and $B$ to - with the approval of a third party $C$ - exchange $m$ units of currency $c_{1}$ for $n$ units of currency $c_{2}$ in a fair manner; i.e., in a way such that if either $A$ or $B$ stops participating at any point in the interaction, the other party loses nothing.

spend transaction requires Alice to present the pre-image $x$ in order to redeem the funds, and his refund transaction can be spent only after some time $t_{2}$ has passed.

$$
\begin{aligned}
& \operatorname{addr} \leftarrow\left\{\begin{array}{cc}
\frac{\text { Spend } \mathrm{T} \times\left(\vec{h}, v, p k_{1}, p k_{2}, p k_{3}\right)}{\text { multiaddr }\left(p k_{1}, p k_{2}, p k_{3}\right)} & \text { if } t>t_{1} \\
p k_{2} & \text { if } H\left(x_{i}\right)=h[i] \forall i
\end{array}\right. \\
& \text { return (addr, } \left.\operatorname{tx}\left(p k_{1} \stackrel{v}{\rightarrow} \mathrm{addr}\right)\right) \\
& \underline{\operatorname{RefundT} \times\left(v, \operatorname{addr}_{\text {in }}, \operatorname{addr}_{\text {out }}\right)} \\
& \text { return } \mathrm{tx}\left(\mathrm{addr}_{\text {in }} \stackrel{v}{\rightarrow} \operatorname{addr}_{\text {out }}\right)
\end{aligned}
$$

Alice begins by creating her spend and refund transactions, as well as picking the value $x$ and computing $H(x)$. She then "commits" to the currency $c_{2}$ being traded with using a second hash $h_{2}$ and sends the refund transaction, signed by herself, to
Carol. If Carol is satisfied with the proposed exchange, she can sign the transaction and give this signature to Alice. Alice now solicits a signature from Bob; once she has signatures from both Bob and Carol, she now has a transaction that she can use to refund her currency after time $t_{1}$ has passed. Thus, it is safe for her to publish the spend transaction in the blockchain for $c_{1}$. Bob then follows suit by creating his own spend and refund transactions, soliciting signatures from Alice and Carol, and publishing his spend transaction once he has a valid refund transaction that he can use if necessary.

Once both transactions are accepted into their respective blockchains, Alice - who so far is the only one with knowledge of the pre-image $x$-can redeem the $n$ units of currency $c_{2}$ using Bob's spend transaction; in doing so, she implicitly reveals $x$. Thus, Bob can now redeem the $m$ units of currency $c_{1}$ using Alice's spend transaction and the exchange is complete. If Alice does not redeem Bob's spend transaction, then after time $t_{2}$ Bob can use his refund transaction to redeem the currency himself (so it is important that $t_{2}<t_{1}$ ). 